\title{
Cooperative overlay secondary transmissions exploiting primary retransmissions
}

\author{
Samuel Baraldi Mafra ${ }^{1 *}$, Richard Demo Souza ${ }^{1}$, João Luiz Rebelatto ${ }^{1}$, Evelio MG Fernandez² \\ and Hirley Alves ${ }^{1,3}$
}

\begin{abstract}
We introduce an overlay cooperative cognitive radio scheme, in which the secondary network transmits only during the retransmissions of the primary network. The secondary network makes use of multiple relays in order to increase its performance compared to a non-cooperative scenario. Moreover, the secondary operates without harming the performance of the primary network. Different cooperative protocols are employed and associated with hybrid automatic repeat request mechanisms. Our results show that the incremental decode-and-forward technique allows the secondary network to achieve the highest throughput among the considered methods, at the cost of a very small degradation in the performance of the primary network.
\end{abstract}

\section{Introduction}

Cognitive radio was introduced by Mitola and Maguire [1] to designate adaptive and intelligent communication devices, which can learn about its surroundings. Later, Haykin [2] defined cognitive radio as an intelligent wireless communication system able to adapt certain parameters (such as transmit power, carrier frequency, etc.) in order to provide highly reliable communications and efficient utilization of the radio spectrum. Furthermore, cognitive radio protocols can be divided into interweave, underlay, and overlay [3,4]. In the interweave protocol, the unlicensed users (also referred to as secondary users) monitor the radio spectrum and communicate over spectrum holes without causing interference to the licensed users (or primary users). In the underlay protocol, the secondary users are allowed to transmit simultaneously to the primary users whereas the interference they cause is below a given threshold [5-7].

In the overlay cognitive radio protocol, the secondary users know, a priori, the primary user message. With this knowledge and using advanced signal processing techniques $[8,9]$, the secondary can transmit concurrently with the primary, without considerably harming its performance [4]. In [10], the authors proposed an overlay

\footnotetext{
*Correspondence: mafrasamuel@gmail.com

${ }^{1}$ Federal University of Technology - Paraná (UTFPR), Curitiba, 80230-901

Paraná, Brazil

Full list of author information is available at the end of the article
}

cognitive radio protocol in which the secondary exploits the primary retransmissions. The proposal in [10] is based on the fact that in many cases, there is an excess of mutual information after a retransmission with respect to the minimum mutual information required by the primary receiver to correctly decode the message. Then, during the retransmission, the primary link can tolerate a certain amount of interference without losing performance. Nevertheless, it is still of importance that this interference is kept to a minimum, confined to the excess mutual information in the primary link. However, in [10], the secondary interference may exceed the limit imposed by the excess of mutual information in the primary link, and the authors proposed a solution to eliminate this interference which requires global channel knowledge at the secondary transmitter (secondary-secondary, secondaryprimary, and primary-primary channels). However, such global channel knowledge is much difficult to be obtained in practice. In [11], it is considered a similar scenario, but assuming that the nodes in the secondary network are provided with multiple antennas, which enables to considerably decrease the interference on the primary, without the need of global channel knowledge. However, this strategy may not be applied in situations where the size or cost of the devices limit the installation of multiple antennas.

An alternative to multiple antennas is to consider cooperative communications [12-14], where one or more

\section{焦 Springer}

D 2013 Mafra et al: licensee Springer. This is an Open Access article distributed under the terms of the Creative Commons Attribution License (http://creativecommons.org/licenses/by/2.0), which permits unrestricted use, distribution, and reproduction in any medium, provided the original work is properly cited. 
nodes help the communication between source and destination by acting as relays, achieving spatial diversity even in a network composed of single antenna devices. In [12], the authors introduce the cooperative decodeand-forward (DF) protocol and its selective (SDF) and incremental (IDF) variants. In the SDF protocol, the message is forwarded only if its decoding at the relay was successful. Finally, in the IDF protocol, similarly to the SDF protocol, the message also needs to be correctly decoded by the relay; however, the forwarding occurs only when requested by the destination. The higher the number of relays available for cooperation, the higher is the performance of the aforementioned cooperative protocols if an appropriate strategy is adopted such as best relay selection [15].

\subsection{Contribution}

We consider the same overlay cognitive radio scenario as in $[10,11]$, but including a cooperative secondary network with multiple relays, while the methods in $[10,11]$ consider a non-cooperative secondary network. We investigate the performance of both the primary and the secondary networks in terms of throughput. In our proposed scheme, we consider the use of the SDF and IDF protocols, where the secondary destination combines the messages received from the secondary transmitter and from the relay by means of maximal ratio combining (MRC). In this work, our aim is to show that, while considering a cognitive radio protocol as that in [10], the proposed cooperative secondary network exploiting primary retransmissions is able to transmit at non-negligible rates while causing practically no harm to the primary communications, without requiring global CSI. Moreover, the proposed cooperative secondary network is shown, through numerical and analytical results, to perform considerably better in terms of achievable rate as well as in terms of protecting the primary communications than the non-cooperative secondary network proposed in [10].

The remainder of this paper is organized as follows. Section 2 describes the system model. The proposed scheme is introduced and analysed in Section 3. Section 4 presents some analytical and numerical results, while Section 5 concludes the paper.

\section{System model}

We consider a primary network composed of a transmitter $T_{p}$ and a destination $D_{p}$. The secondary network consists of a secondary transmitter $T_{s}$, a secondary destination $D_{s}$, and $N$ potentially cooperating relays denoted as $r(l)$, with $l \in \Lambda=\{1,2, \ldots, N\}$. The $N$ relays are considered to be in a cluster, so that they are assumed to be at approximately the same position. The simplifying assumption that the relays are organized within a cluster is commonly utilized in the literature and can represent a number of practical scenarios (please see, for instance, [16-20]). The channel between any transmitter $i$ and receiver $j$ is denoted by $h_{i j}$ and follows a Nakagami- $m$ distribution [21] with fading parameter $m_{i j}$ and average power $\lambda_{i j}$. The Nakagami$m$ distribution is a general approach that includes the Rayleigh distribution as a special case (when $m=1$ ). Moreover, through this model, the severity of the fading can be adjusted by the parameter $m$. Based on experimental results reported in [22], in this paper, we consider values of $m=1$ and $m=2$ for characterizing non-line-of-sight (NLOS) and some line-of-sight (LOS) scenarios.

In our notation $i, j \in\{p, s, r(l)\}$, where $p$ represents the primary transmitter or receiver, $s$ the secondary transmitter or receiver and $r(l)$ the $l$-th relay. The average power is defined as $\lambda_{i j}=\frac{1}{\left(d n_{i j}\right)^{\alpha}}$, where $d n_{i j}=\frac{d_{i j}}{d_{p p}}$ is the normalized distance between transmitter $i$ and receiver $j$ with respect to the distance between $T_{p}$ and $D_{p}\left(d_{p p}\right), d_{i j}$ is the actual distance between transmitter $i$ and receiver $j$, and $\alpha$ is the path-loss exponent. The secondary network operates at the same frequency band and time slot allocated to the primary network. As in [10], we assume that the primary network employs a hybrid automatic repeat request strategy and that the channels are quasi-static. Moreover, as in $[10,11]$, due to delay constraints, we assume that the primary network is allowed to perform only one retransmission. Finally, Figure 1 illustrates the system model, including $T_{p}, D_{p}, T_{s}, D_{s}$, and the selected relay $r$.

Further, the received signal at node $j$ can be written as

$$
y_{i j}=\sqrt{P_{i}} h_{i j} x_{i}+z_{j},
$$

where $P_{i}$ is the transmit power, $x_{i}$ is the transmitted message, and $z_{j}$ is additive white Gaussian noise with variance $\frac{N_{0}}{2}$ per dimension, where $N_{0}$ is the unilateral noise power spectral density, which is assumed to be $N_{0}=1 \mathrm{~W} / \mathrm{Hz}$.

The analysis that follows is based on the throughput, which is defined as the rate of error-free information transfer, and it is determined as a function of the outage probability. The outage probability is the probability that a failure occurs in the communication between nodes $i$ and $j$ [23]. Moreover, an outage can be defined as the event that the mutual information is less than the attempted information rate $R$. For instance, assuming a unitary bandwidth, complex Gaussian channel inputs, a given link with channel realization $h$, transmit power $P$, and in the absence of interference, the mutual information is $I=\log _{2}\left(1+|h|^{2} P\right)$ and the outage probability $\mathcal{O}$ is given by [12]

$$
\mathcal{O}=\operatorname{Pr}\{I<R\}
$$

where $\operatorname{Pr}\{a\}$ is the probability of event $a$. The above outage probability formulation is based on the Shannon limit and is defined for an infinite block length code. However, this assumption does not invalidate our analysis, since it 


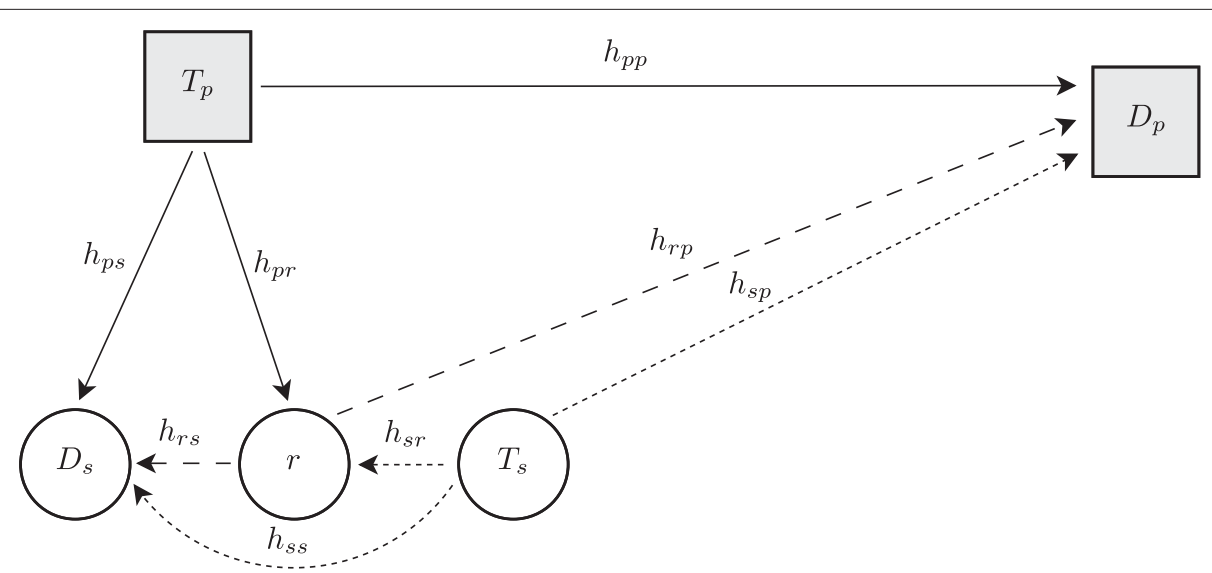

Figure 1 System model with a primary transmitter $T_{p}$, primary destination $D_{p}$, secondary transmitter $T_{s}$, selected relay $r$, and secondary destination $D_{s}$.

has been shown that the outage probability predicts surprisingly well the frame error rate of good practical codes with relatively short block lengths [24,25]. For instance, in the case of a single transmission, the throughput can be written as:

$$
\mathcal{T}=R(1-\mathcal{O}) \quad[\text { bits/channel use }]
$$

\section{Proposed scheme}

The proposed overlay cooperative cognitive radio scheme is based on the exploitation of retransmissions from the primary network. If a given primary transmission fails and the primary receiver requests a retransmission, then it is very likely that after a second transmission from the primary, the accumulated mutual information seen at the primary receiver is above the attempted primary rate, so that there may be an 'excess of mutual information' in the primary link [10]. If the attempted primary rate is $R_{p}$ and the accumulated mutual information at the primary receiver after a retransmission is $I_{p, 2}>R_{p}$, then the excess of mutual information is $I_{p, 2}-R_{p}$. This means that the primary network could tolerate some amount of interference without losing performance, therefore providing a margin for the secondary network operation.

Moreover, we assume that the secondary network only operates during a primary retransmission if $D_{s}$ was able to decode the original primary transmission from $T_{p}$. That is because in this case, $D_{s}$ would be able to remove the primary interference during the primary retransmission, without requiring $T_{s}$ to make use of complex transmission techniques, such as dirty paper coding [26], nor requiring global channel knowledge. Note also that $D_{s}$ must inform $T_{s}$ that it could decode the original primary transmission, so that $T_{s}$ becomes aware of the secondary transmission opportunity. This can be done either through the main channel or through a dedicated low rate control channel.
As we assume a cooperative secondary network, we consider a reactive relay selection scheme [15], so that the cooperating relay is selected in a distributed way after a transmission from $T_{s}$. Let $\Phi \subset \Lambda$ be a set containing the indexes of the relays that could correctly decode both the messages transmitted by $T_{p}$ and $T_{s}$. We consider that the selected cooperating relay, $r\left(l^{*}\right)$, is chosen as:

$$
l^{*}=\underset{l \in \Phi}{\arg \max }\left|h_{r(l) s}\right|^{2},
$$

i.e., the relay chosen to cooperate will be the one with the best channel condition with respect to $D_{s}$ among those that could decode the messages from both $T_{p}$ and $T_{s}$. Hereafter, we will refer to the selected relay only as $r$. Note that the selected relay is chosen based only on the quality of the link between the relay and the destination. That is sufficient, as the relay is selected, only among those relays that were able to decode the source message.

A practical way to choose the best relay is to make each relay wait before transmitting for a time inversely proportional to its instantaneous channel state with respect to $D_{s}$. Thus, in order to avoid collisions, the relay with the best condition will be the first to transmit while the others remain silent when perceiving a busy channel [15]. If none relay decoded the messages from $T_{p}$ and $T_{s}$, then all relays remain silent. Additionally, since $r$ could decode the original primary transmission, then it can eliminate the primary interference during the retransmission. Finally, as we consider a cooperative secondary network based on half-duplex nodes, the secondary transmissions occur in two time slots. As the secondary network only operates during the retransmission of the primary network, each time slot in the secondary network has half the duration of a time slot in the primary network. 
In summary, the operation of the proposed cooperative cognitive network is as follows:

- The primary transmitter $T_{p}$ sends a new message to the primary receiver $D_{p}$;

- If the message was successfully received, then $D_{p}$ sends back an ACK signal to $T_{p}$, otherwise a NACK is sent back;

- In case a NACK is sent by $D_{p}$, then the secondary receiver $D_{s}$ informs the secondary transmitter $T_{s}$ if it could decode the primary message or not;

- If $D_{s}$ decoded the primary message, then a transmission opportunity is opened for the secondary transmitter $T_{s}$, which then transmits concurrently to $T_{p}$ during the primary retransmission;

- The transmission from $T_{s}$ lasts for only half the duration of the primary retransmission. During the second half of the primary retransmission, the selected relay $r$ may forward or not to $D_{s}$ the message sent by $T_{s}$;

- If the secondary network is operating under the SDF protocol, then $r$ only forwards the message to $D_{s}$ if it could decode the message;

- If the secondary network is operating under the IDF protocol, then $r$ only forwards the message to $D_{s}$ if it could decode the message and if $D_{s}$ requested so. If not requested, then $T_{s}$ can send a new message in the second half of the primary time slot.

In what follows, we derive the throughput of the primary and secondary networks considering the proposed scheme.

\subsection{Primary throughput}

First, let us define $\mathcal{O}_{p, 1}$ as the outage probability in the primary link after the first transmission from $T_{p}$. During the first primary transmission, the secondary network is inactive, so that there is no interference at $D_{p}$, and therefore,

$$
\begin{aligned}
\mathcal{O}_{p, 1} & =\operatorname{Pr}\left\{I_{p, 1}<R_{p}\right\}=\operatorname{Pr}\left\{\log _{2}\left(1+\left|h_{p p}\right|^{2} P_{p}\right)<R_{p}\right\} \\
& =\frac{1}{\Gamma\left(m_{p p}\right)} \gamma\left(m_{p p}, \frac{m_{p p}\left(2^{R_{p}}-1\right)}{\lambda_{p p} P_{p}}\right),
\end{aligned}
$$

where $\gamma(a, b)=\int_{0}^{b} y^{a-1} \exp (-y) d y$ and $\Gamma(a)=\int_{0}^{\infty} y^{a-1}$ $\exp (-y) d y$ are, respectively, the lower incomplete Gamma function and the complete Gamma function $[27,28]$.

Recall that, as in $[10,11]$, we assume that the primary network is allowed to perform a retransmission when the first transmission fails. Thus, an outage occurs in the primary network when the accumulated mutual information after at most two transmissions is less than the attempted rate $R_{p}$. Moreover, in order to define the overall outage probability of the primary network, we first define $U_{n}$, the event that no relay was able to correctly decode both the messages from $T_{p}$ and $T_{s}$, so that if the secondary link is active, then it operates in a non-cooperative mode. Then, the outage probability in the primary link after a retransmission, $\mathcal{O}_{p, 2}$, can be written as

$$
\begin{aligned}
\mathcal{O}_{p, 2}= & \underbrace{\operatorname{Pr}\left\{I_{p, 2}<R_{p} \mid \overline{\mathcal{O}_{p s}}, \overline{U_{n}}\right\} \cdot \overline{\mathcal{O}_{p s}} \cdot \operatorname{Pr}\left\{\overline{U_{n}}\right\}}_{(\mathrm{A})} \\
& +\underbrace{\operatorname{Pr}\left\{I_{p, 2}<R_{p} \mid \overline{\mathcal{O}_{p s}}, U_{n}\right\} \cdot \overline{\mathcal{O}_{p s}} \cdot \operatorname{Pr}\left\{U_{n}\right\}}_{(\mathrm{B})} \\
& +\underbrace{\operatorname{Pr}\left\{I_{p, 2}<R_{p} \mid \mathcal{O}_{p s}\right\} \cdot \mathcal{O}_{p s}}_{(\mathrm{C})} .
\end{aligned}
$$

where

$$
\begin{aligned}
\mathcal{O}_{p s} & =\operatorname{Pr}\left\{I_{p s}<R_{p}\right\}=\operatorname{Pr}\left\{\log _{2}\left(1+\left|h_{p s}\right|^{2} P_{p}\right)<R_{p}\right\} \\
& =\frac{1}{\Gamma\left(m_{p s}\right)} \gamma\left(m_{p s}, \frac{m_{p s}\left(2^{R_{p}}-1\right)}{\lambda_{p s} P_{p}}\right)
\end{aligned}
$$

is the outage probability in the link between the primary transmitter $T_{p}$ and the secondary receiver $D_{s}$. Therefore, $\overline{\mathcal{O}_{p s}}=1-\mathcal{O}_{p s}$ is the probability that $D_{s}$ could decode the message sent by $T_{p}$ after its first transmission. The probability of event $U_{n}$ is given by

$$
\begin{aligned}
\operatorname{Pr}\left\{U_{n}\right\}= & {\left[\frac{\gamma\left(m_{p r(l)}, \frac{m_{p r(l)}\left(2^{R_{p}}-1\right)}{\lambda_{p r(l)} P_{p}}\right)}{\Gamma\left(m_{\operatorname{pr}(l)}\right)}+\frac{\gamma\left(m_{s r(l)}, \frac{m_{s r(l)}\left(2^{R_{s}}-1\right)}{\lambda_{s r(l)} P_{s}}\right)}{\Gamma\left(m_{s r(l)}\right)}\right.} \\
& \left.-\frac{\gamma\left(m_{s r(l)}, \frac{m_{s r(l)}\left(2^{R_{s}}-1\right)}{\lambda_{s r(l)} P_{s}}\right)}{\Gamma\left(m_{s r(l)}\right)} \cdot \frac{\gamma\left(m_{\operatorname{pr}(l)}, \frac{m_{p r(l)}\left(2^{R_{p}}-1\right)}{\lambda_{p r(l)} P_{p}}\right)}{\Gamma\left(m_{p r(l)}\right)}\right]^{N},
\end{aligned}
$$

and the derivation is detailed in Appendix 1. Notice that $\operatorname{Pr}\left\{\overline{U_{n}}\right\}=1-\operatorname{Pr}\left\{U_{n}\right\}$

The term (A) in Eq. (6) is the probability that $D_{p}$ did not decode the message transmitted by $T_{p}$ after the retransmission, given that $D_{s}$ correctly decoded the primary message and at least one relay decoded both primary and secondary messages. The term (B) in Eq. (6) represents the probability that $D_{p}$ did not recover the message transmitted by $T_{p}$ after the retransmission, given that $D_{s}$ correctly decoded the primary message but no relay was able to decode both $T_{p}$ and $T_{s}$ messages, so that the secondary network operates in a non-cooperative fashion. Finally, the term (C) in Eq. (6) is the probability that an outage occurred in the primary link after two transmissions, given that $D_{s}$ failed to decode the message from $T_{p}$.

In order to analytically evaluate the outage probability in the primary link after a retransmission, it is important to note that the mutual information $I_{p, 2}$ seen at the 
primary destination $D_{p}$ after two transmissions from the primary depends on the behavior of the secondary network. For instance, if the secondary network is not active, the mutual information is $I_{p, 2}=2 \log _{2}\left(1+\left|h_{p p}\right|^{2} P_{p}\right)$, so that the primary destination $D_{p}$ does not suffer any interference from the secondary transmitter or relays. If the secondary network is active and at least one of the $N$ available relays could decode both the messages sent by $T_{p}$ and $T_{s}$, there will be interference at $D_{p}$ and the mutual information is $I_{p, 2}=\log _{2}\left(1+\left|h_{p p}\right|^{2} P_{p}\right)+$ $\frac{1}{2} \log _{2}\left(1+\frac{\left|h_{p p}\right|^{2} P_{p}}{1+\left|h_{s p}\right|^{2} P_{s}}\right)+\frac{1}{2} \log _{2}\left(1+\frac{\left|h_{p p}\right|^{2} P_{p}}{1+\left|h_{r p}\right|^{2} P_{r}}\right)$. Note that the first term corresponds to the first transmission, while the second and third terms correspond to the retransmission which include interference from $T_{s}$ and from the selected cooperating relay $r$, respectively. Moreover, in the case of event $U_{n}$, in which none relay was able to decode both the messages from $T_{p}$ and $T_{s}$, then $I_{p, 2}=\log _{2}(1+$ $\left.\left|h_{p p}\right|^{2} P_{p}\right)+\frac{1}{2} \log _{2}\left(1+\frac{\left|h_{p p}\right|^{2} P_{p}}{1+\left|h_{s p}\right|^{2} P_{s}}\right)+\frac{1}{2} \log _{2}\left(1+\left|h_{p p}\right|^{2} P_{p}\right)$. Again, the first part of the mutual information corresponds to the original transmission. Note that since all the relays remain silent during the retransmission, there is only interference from $T_{s}$, which occurs only during half of the primary retransmission, so that during half of the retransmission, there is no interference at $D_{p}$. Finally, considering all the above, the outage probability in the primary link after a retransmission, $O_{p, 2}$, can be written in closed form as:

$$
\begin{aligned}
\mathcal{O}_{p, 2}= & \frac{\gamma\left(m_{p p}, m_{p p} \frac{\sqrt{\left(1+\sigma_{p}^{2}\right)^{2}+4 \sigma_{p}^{2} \cdot\left(2^{R_{p}}-1\right)}-1-\sigma_{p}^{2}}{2 P_{p} \lambda_{p p}}\right)}{\Gamma\left(m_{p p}\right)} \\
& \cdot\left(1-\frac{\gamma\left(m_{p s}, \frac{m_{p s}\left(2^{R_{p}}-1\right)}{\lambda_{p s} P_{p}}\right)}{\Gamma\left(m_{p s}\right)}\right) \\
& +\frac{\gamma\left(m_{p p}, \frac{m_{p p}\left(2^{\frac{1}{2} R_{p}}-1\right)}{\lambda_{p p} P_{p}}\right)}{\Gamma\left(m_{p p}\right)} \cdot \frac{\gamma\left(m_{p s}, \frac{m_{p s}\left(2^{R_{p}}-1\right)}{\lambda_{p s} P_{p}}\right)}{\Gamma\left(m_{p s}\right)} .
\end{aligned}
$$

Refer to Appendix 2 for further details.

Moreover, based on the above definitions, we can write the throughput of the primary in the presence of the secondary cooperative network as

$$
\begin{aligned}
\mathcal{T}_{p} & =R_{p}\left(1-\mathcal{O}_{p, 1}\right)+\frac{R_{p}}{2} \mathcal{O}_{p, 1} \operatorname{Pr}\left\{I_{p, 2}>R_{p} \mid I_{p, 1}<R_{p}\right\} \\
& =R_{p}\left(1-\mathcal{O}_{p, 1}\right)+\frac{R_{p}}{2} \mathcal{O}_{p, 1}\left(1-\frac{\mathcal{O}_{p, 2}}{\mathcal{O}_{p, 1}}\right) \\
& =R_{p}\left(1-\mathcal{O}_{p, 1}\right)+\frac{R_{p}}{2}\left(\mathcal{O}_{p, 1}-\mathcal{O}_{p, 2}\right)
\end{aligned}
$$

in which we used Bayes' theorem and the fact that $I_{p, 2} \geq I_{p, 1}$.

\subsection{Secondary throughput}

Now, we analyze the throughput of the secondary network using both SDF and IDF protocols. We assume that $r$ forwards the message transmitted by $T_{s}$. Upon receiving two copies of the same message (from $T_{s}$ and $r$ ), we consider that $D_{s}$ performs MRC. Recall that the secondary network only operates if the initial primary transmission failed and if the secondary transmitter was able to decode the primary message.

When using the SDF protocol, the throughput of the secondary network when the attempted rate is $R_{s}$ can be written as

$$
\begin{aligned}
\mathcal{T}_{\mathrm{SDF}} & =\underbrace{\frac{R_{s}}{2} \mathcal{O}_{p, 1} \overline{\mathcal{O}_{p s}} \overline{\mathcal{O}_{s s}}}_{\mathrm{A}}+\underbrace{\frac{R_{s}}{2} \mathcal{O}_{p, 1} \overline{\mathcal{O}_{p s}} \operatorname{Pr}\left\{\overline{U_{n}}\right\} \mathcal{O}_{s s}\left(1-\frac{\mathcal{O}_{\mathrm{MRC}}}{\mathcal{O}_{s s}}\right)}_{\mathrm{B}} \\
& =\frac{R_{s}}{2} \mathcal{O}_{p, 1} \overline{\mathcal{O}_{p s}} \overline{\mathcal{O}_{s s}}+\frac{R_{s}}{2} \mathcal{O}_{p, 1} \overline{\mathcal{O}_{p s}} \operatorname{Pr}\left\{\overline{U_{n}}\right\}\left(\mathcal{O}_{s s}-\mathcal{O}_{\mathrm{MRC}},\right.
\end{aligned}
$$

where $\mathcal{O}_{s s}$ is the outage probability in the link between $T_{s}$ and $D_{s}$ and it is defined as

$$
\begin{aligned}
\mathcal{O}_{s s} & =\operatorname{Pr}\left\{I_{s s}<R_{s}\right\}=\operatorname{Pr}\left\{\log _{2}\left(1+\left|h_{s s}\right|^{2} P_{s}\right)<R_{s}\right\} \\
& =\frac{\gamma\left(m_{s s}, \frac{m_{s s}\left(2^{R_{s}}-1\right)}{\lambda_{s s} P_{s}}\right)}{\Gamma\left(m_{s s}\right)}
\end{aligned}
$$

while

$$
\begin{aligned}
\mathcal{O}_{\mathrm{MRC}} & =\operatorname{Pr}\left\{I_{\mathrm{MRC}}<R_{s}\right\} \\
& =\operatorname{Pr}\left\{\log _{2}\left(1+\left(\left|h_{s s}\right|^{2}+\left|h_{r s}\right|^{2}\right) P_{s}\right)<R_{s}\right\}
\end{aligned}
$$

corresponds to the outage probability at $D_{s}$ given that $T_{s}$ and $r$ cooperate and that their messages are combined at the destination. Closed-form outage probability expressions for Eq. (13) are given in Appendix 3 for two different cases, when there is some LOS $(m=2)$ and under NLOS condition $(m=1)$.

The fragment (A) in Eq. (11) refers to the case where the secondary message is successfully delivered over the secondary direct link between $T_{s}$ and $D_{s}$. The product $\mathcal{O}_{p, 1} \overline{\mathcal{O}_{p s}}$ accounts for the fact that there must have happened an outage in the previous primary transmissions $\left(\mathcal{O}_{p, 1}\right)$ and that $D_{s}$ was able to decode the primary message $\left(\overline{\mathcal{O}_{p s}}\right)$. The term $\overline{\mathcal{O}_{s s}}$ is the probability that the secondary direct link is not in outage. Moreover, the fragment (B) of Eq. (11) considers the case in which the secondary direct link is in outage but the secondary cooperative link is not. Again, the terms $\mathcal{O}_{p, 1} \overline{\mathcal{O}_{p s}}$ are the probability that the primary link was in outage in the previous transmission and that $D_{s}$ was able to decode the source message. As already mentioned, the term $\mathcal{O}_{s s}$ is the probability that the secondary direct link (between $T_{s}$ and $D_{s}$ ) is in outage, while $\operatorname{Pr}\left\{\overline{U_{n}}\right\}$ is the probability that at least one relay is able to cooperate. The expression $\left(1-\frac{\mathcal{O}_{M R C}}{\mathcal{O}_{s s}}\right)$ is the probability 
that the secondary cooperative link (the one formed by the combination of the transmissions from $T_{s}$ and from the selected relay $r$ ) is not in outage given that the direct link is in outage. Finally, the maximum achievable throughput at the secondary network using the SDF protocol is $\frac{R_{s}}{2}$, since the message is transmitted using two time slots.

Very similar to the SDF case, when using the IDF protocol, we have that

$$
\mathcal{T}_{\mathrm{IDF}}=R_{s} \mathcal{O}_{p, 1} \overline{\mathcal{O}_{p s}} \overline{\mathcal{O}_{s s}}+\frac{R_{s}}{2} \mathcal{O}_{p, 1} \overline{\mathcal{O}_{p s}} \operatorname{Pr}\left\{\overline{U_{n}}\right\}\left(\mathcal{O}_{s s}-\mathcal{O}_{\mathrm{MRC}}\right)
$$

Note that in this case, the only difference is that the maximum throughput in the secondary network is $R_{s}$, since the selected relay only cooperates if requested by $D_{s}$, so that it is possible to deliver a message in a single time slot if the secondary direct link is not in outage.

\section{Numerical results}

This section presents some numerical results in order to investigate the performance of the proposed cognitive cooperative scheme. Monte Carlo simulations are represented using black square markers and are included to demonstrate the accuracy of the analytical derivations. Moreover, we consider a path-loss exponent of $\alpha=4$, $d_{s p} \approx d_{r p}, d_{p s} \approx d_{p r}$, and $d_{s r}=d_{s s} / 2$ (relay is positioned halfway between $T_{s}$ and $D_{s}$ ). The distances in the network are normalized with respect to $d_{p p}=1$. Finally, we assume that $T_{s}$ and $r$ transmit with the same power $P_{s}$.

Figure 2 evaluates the throughput versus the attempted rate $R_{s}$. We compare the proposed cooperative method to the non-cooperative scheme in [10], considering only one relay. The other parameters of interest are $R_{p}=4$ bits per channel use (bpcu), $P_{s}=-5 \mathrm{~dB}, P_{p}=10 \mathrm{~dB}, \lambda_{p p}=\lambda_{s p}=$ $\lambda_{r p}=1, \lambda_{p s}=\lambda_{p r}=2^{4}, \lambda_{s s}=2^{4}, \lambda_{s r}=\lambda_{r s}=4^{4}, m_{i j}=1$ (NLOS) $\forall i, j$.

From Figure 2, we can see that, without considerably harming the primary network, the IDF-based cooperative scheme achieves a throughput of $1 \mathrm{bpcu}$ for $R_{s}=4 \mathrm{bpcu}$ while the non-cooperative case achieves only $0.15 \mathrm{bpcu}$. In Figure 3, we consider that the nodes of the secondary network are even closer to each other as well as to $T_{p}$, assuming that $\lambda_{p p}=\lambda_{s p}=\lambda_{r p}=1, \lambda_{p s}=\lambda_{p r}=4^{4}, \lambda_{s s}=4^{4}$, $\lambda_{s r}=\lambda_{r s}=8^{4}$. In such a scenario, it is possible for the secondary network to achieve a throughput of $1.9 \mathrm{bpcu}$ when $R_{s}=6 \mathrm{bpcu}$ for the IDF-based cooperative scheme without harming the primary link.

Figure 4 compares the proposed scheme to the method proposed in [10] in terms of throughput versus $P_{s}$, still considering an NLOS scenario. The nodes of the secondary are closer to $T_{p}$ than to $D_{p}\left(\lambda_{p p}=\lambda_{s p}=\lambda_{r p}=1\right.$, $\left.\lambda_{p s}=\lambda_{p r}=2^{4}, \lambda_{s s}=2^{4}, \lambda_{s r}=\lambda_{r s}=4^{4}\right), R_{p}=3 \mathrm{bpcu}, R_{s}=4$ bpcu, $P_{p}=10 \mathrm{~dB}$, and there is only one available relay.

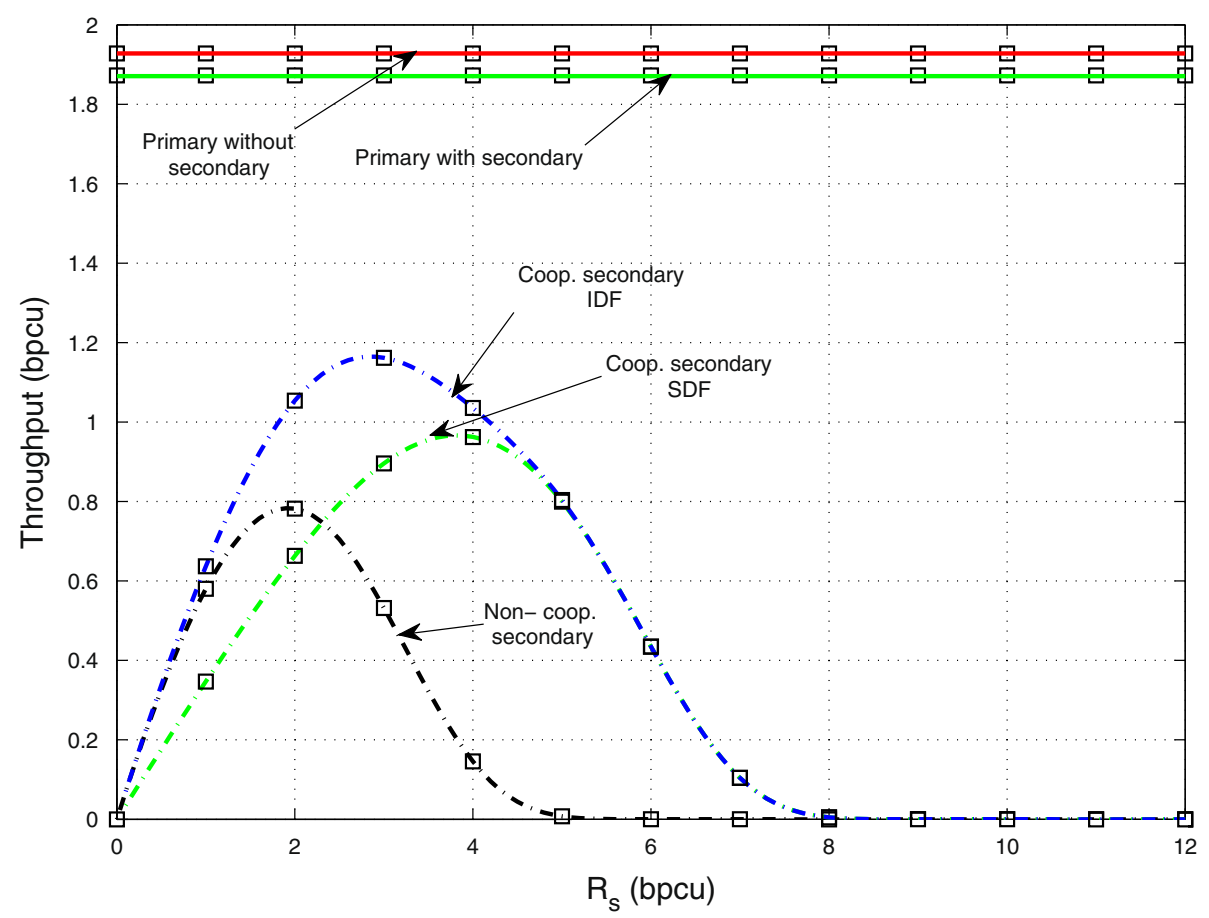

Figure 2 Throughput versus $R_{s}$, for $R_{p}=4 \mathrm{bpcu}, P_{s}=-5 \mathrm{~dB}, P_{p}=10 \mathrm{~dB}, \lambda_{p p}=\lambda_{s p}=\lambda_{r p}=1, \lambda_{p s}=\lambda_{p r}=2^{4}, \lambda_{s s}=2^{4}, \lambda_{s r}=\lambda_{r s}=4^{4}$. The black square marker represents the Monte Carlo simulations. 


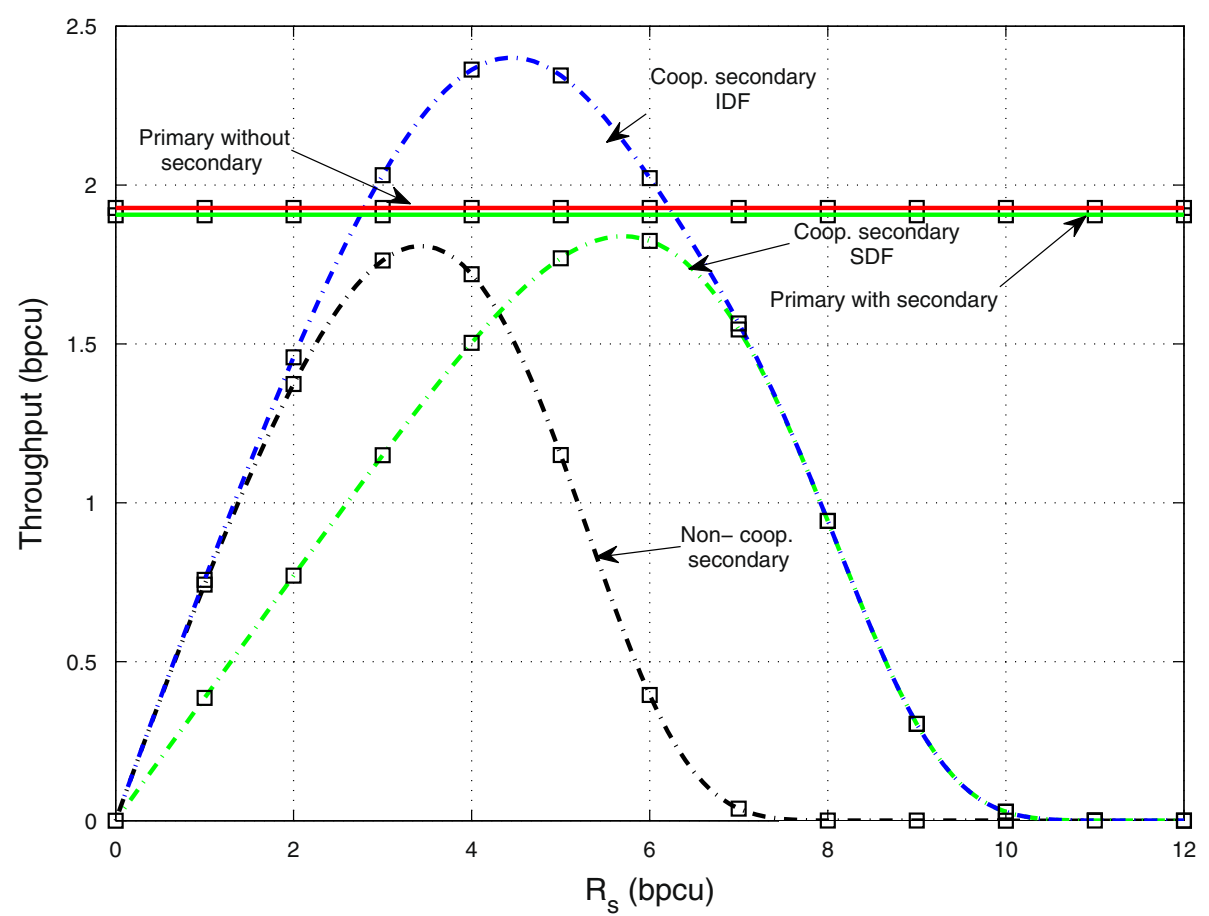

Figure 3 Throughput versus $R_{s}$, for $R_{p}=4 \mathrm{bpcu}, P_{s}=-10 \mathrm{~dB}, P_{p}=10 \mathrm{~dB}, \lambda_{p p}=\lambda_{s p}=\lambda_{r p}=1, \lambda_{p s}=\lambda_{p r}=4^{4}, \lambda_{s s}=4^{4}, \lambda_{s r}=\lambda_{r s}=8^{4}$. The black square marker represents the Monte Carlo simulations.

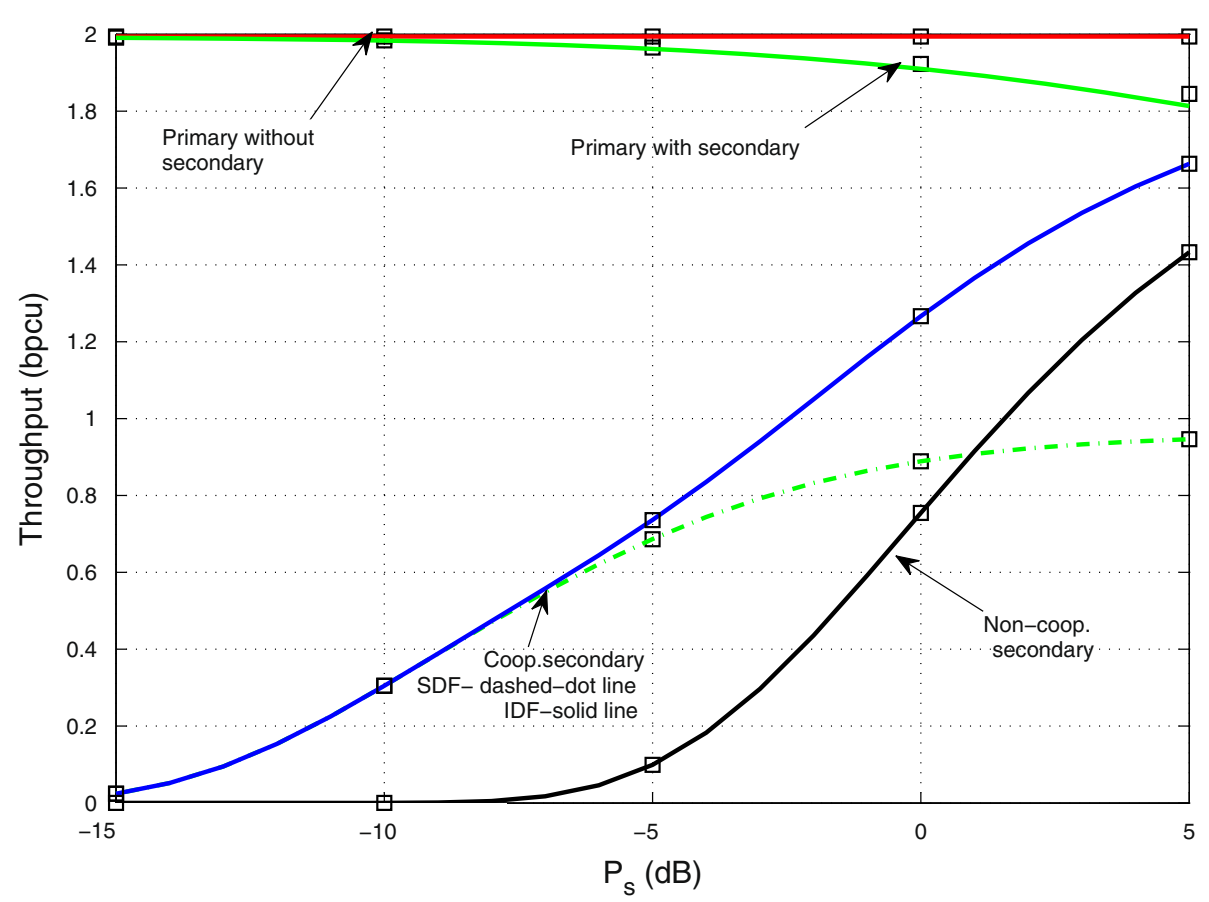

Figure 4 Throughput versus $P_{s}$, for $R_{p}=3 \mathrm{bpcu}, \boldsymbol{R}_{s}=\mathbf{4} \mathrm{bpcu}, \boldsymbol{P}_{p}=10 \mathrm{~dB}, \lambda_{p p}=\lambda_{s p}=\lambda_{r p}=1, \lambda_{p s}=\lambda_{p r}=2^{4}, \lambda_{s s}=2^{4}, \lambda_{s r}=\lambda_{r s}=4^{4}$. The black square marker represents the Monte Carlo simulations. Comparison between the cooperative method and the non-cooperative method. 
It can be seen that the proposed scheme presents a better performance, achieving a throughput of up to 0.55 bpcu without significantly harming the primary. The IDF-based proposed cooperative scheme outperforms the non-cooperative scheme proposed in [10] for the whole range. Since the IDF-based proposed protocol presents a higher throughput than the SDF-based protocol, thus henceforth we only consider the IDF-based scheme.

In Figure 5, we consider the same relative position between nodes as in Figure 4 . The impact of the existence of some LOS in the $T_{p}$ to $R_{s}$ and $T_{p}$ to $D_{s}$ links, as well as in the secondary network, is evaluated by setting $m_{s s}=m_{s r}=m_{r s}=m_{p r}=m_{p s}=2\left(m_{p p}=m_{s p}=m_{r p}=1\right.$ are kept unchanged). The idea behind modifying the $m$ parameter on the Nakagami- $m$ fading distribution relies on the fact that secondary networks is more likely to experience shorter links and some LOS. We also consider that $R_{p}=3 \mathrm{bpcu}, R_{s}=4 \mathrm{bpcu}, P_{p}=10 \mathrm{~dB}$. The performance of the proposed cooperative scheme under NLOS $\left(m_{i j}=1 \forall i, j\right)$ is also shown as a reference. As we can see, the secondary performance increases considerably in the presence of some LOS, specially in the $P_{s}$ range where the impact on the primary performance is negligible. The primary performance is not affected by the existence of some LOS in the $T_{p}$ to $R_{s}$ and $T_{p}$ to $D_{s}$ links.

The impact on the throughput by increasing the number of available relays in the secondary network is evaluated in
Figure 6, considering the same relative position between nodes as in Figures 4 and 5 , and the LOS condition. Moreover, $R_{p}=4 \mathrm{bpcu}, R_{s}=4 \mathrm{bpcu}$, and $P_{p}=10 \mathrm{~dB}$. From Figure 6 , it can be seen that the throughput increases as the number of available relays increases. For $P_{s}=-10$ $\mathrm{dB}$, for example, the secondary link achieves a throughput of $0.72 \mathrm{bpcu}$ with only one relay and $1.08 \mathrm{bpcu}$ with four cooperating relays, with a very little interference on the primary link. Similar analysis is shown in Figure 7, but with the secondary nodes closer to each other and to the primary transmitter, by assuming that $\lambda_{p p}=\lambda_{s p}=\lambda_{r p}=1$, $\lambda_{p s}=\lambda_{p r}=4^{4}, \lambda_{s s}=4^{4}, \lambda_{s r}=\lambda_{r s}=8^{4}$.

From Figure 7, we observe that when $P_{s}=-16 \mathrm{~dB}$, the secondary throughput is increased from 1.06 to $1.60 \mathrm{bpcu}$ when the number of available relays is increased from $N=1$ to $N=4$. In these conditions, the secondary network can even outperform the primary network in terms of throughput.

Finally, it is important to point out that if the secondary network is much closer to the $D_{p}$ than to $T_{p}$, both schemes (the proposed scheme and the one in [10]) do not perform well once the secondary achieves very low throughput and degrades the primary network performance. We recall that as discussed above, the proposed scheme considerably outperforms the method introduced in [10], and even larger gains can be obtained if a larger number of relays is available.

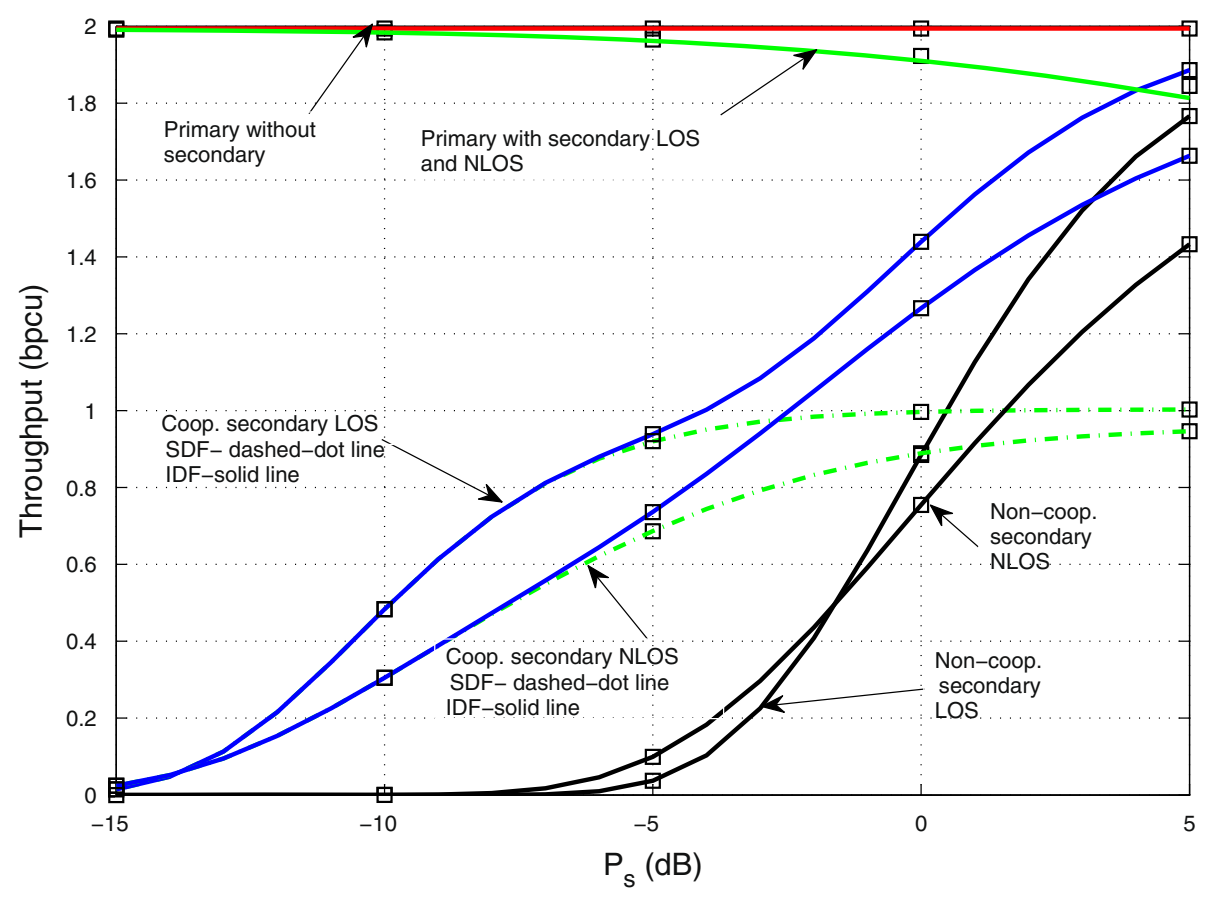

Figure 5 Throughput versus $P_{s}$, for $\boldsymbol{R}_{p}=3 \mathrm{bpcu}, \boldsymbol{R}_{s}=4 \mathrm{bpcu}, \boldsymbol{P}_{p}=10 \mathrm{~dB}, \lambda_{p p}=\lambda_{s p}=\lambda_{r p}=1, \lambda_{p s}=\lambda_{p r}=\mathbf{2}^{\mathbf{4}}, \lambda_{s s}=\mathbf{2}^{\mathbf{4}}, \lambda_{s r}=\lambda_{r s}=4^{\mathbf{4}}$. The black square marker represents the Monte Carlo simulations. Both LOS and NLOS cases are considered. 


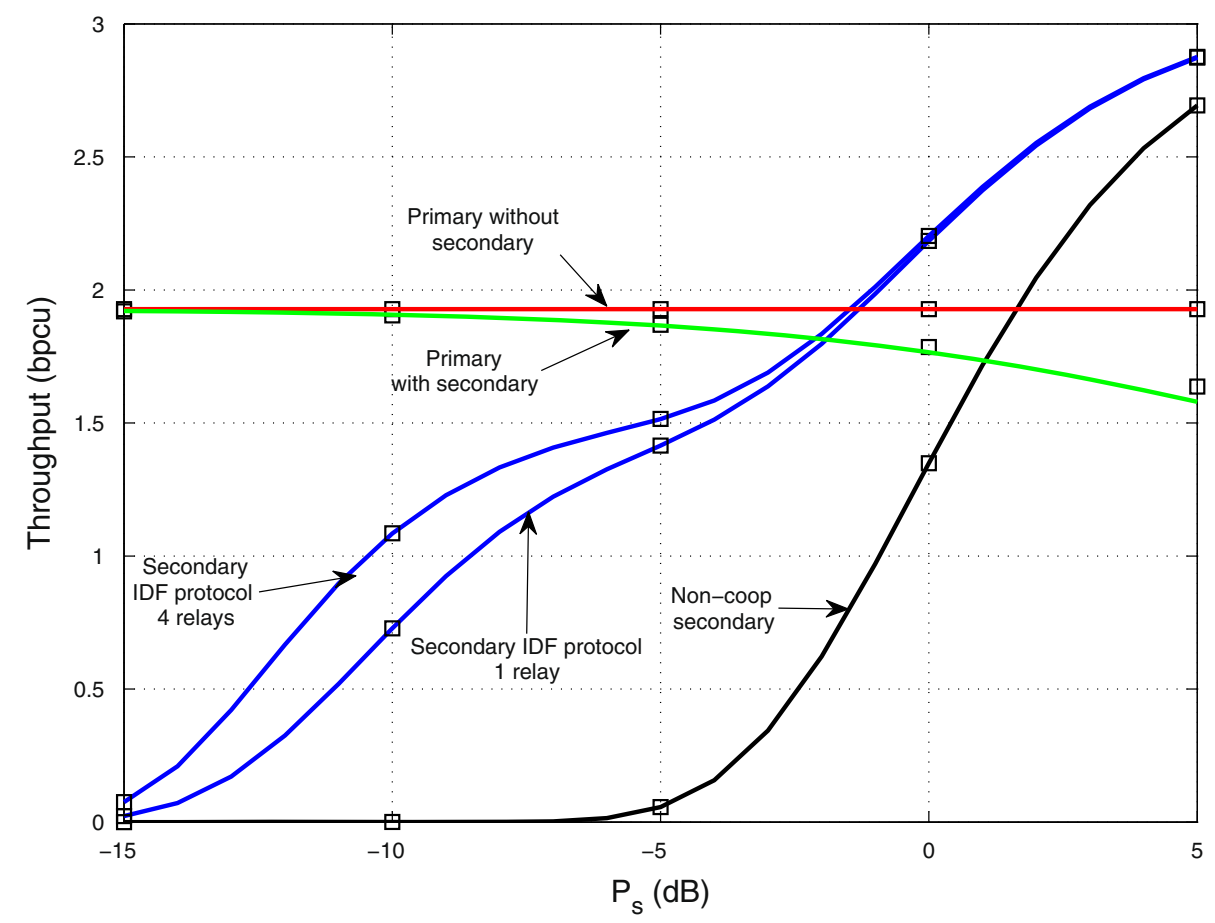

Figure 6 Throughput versus $P_{s}$, for $R_{p}=4 \mathrm{bpcu}, R_{s}=4 \mathrm{bpcu}, P_{p}=10 \mathrm{~dB}, \lambda_{p p}=\lambda_{s p}=\lambda_{r p}=1, \lambda_{p s}=\lambda_{p r}=2^{4}, \lambda_{s s}=2^{4}, \lambda_{s r}=\lambda_{r s}=4^{4}$, considering one and four available relays. The black square marker represents the Monte Carlo simulations.

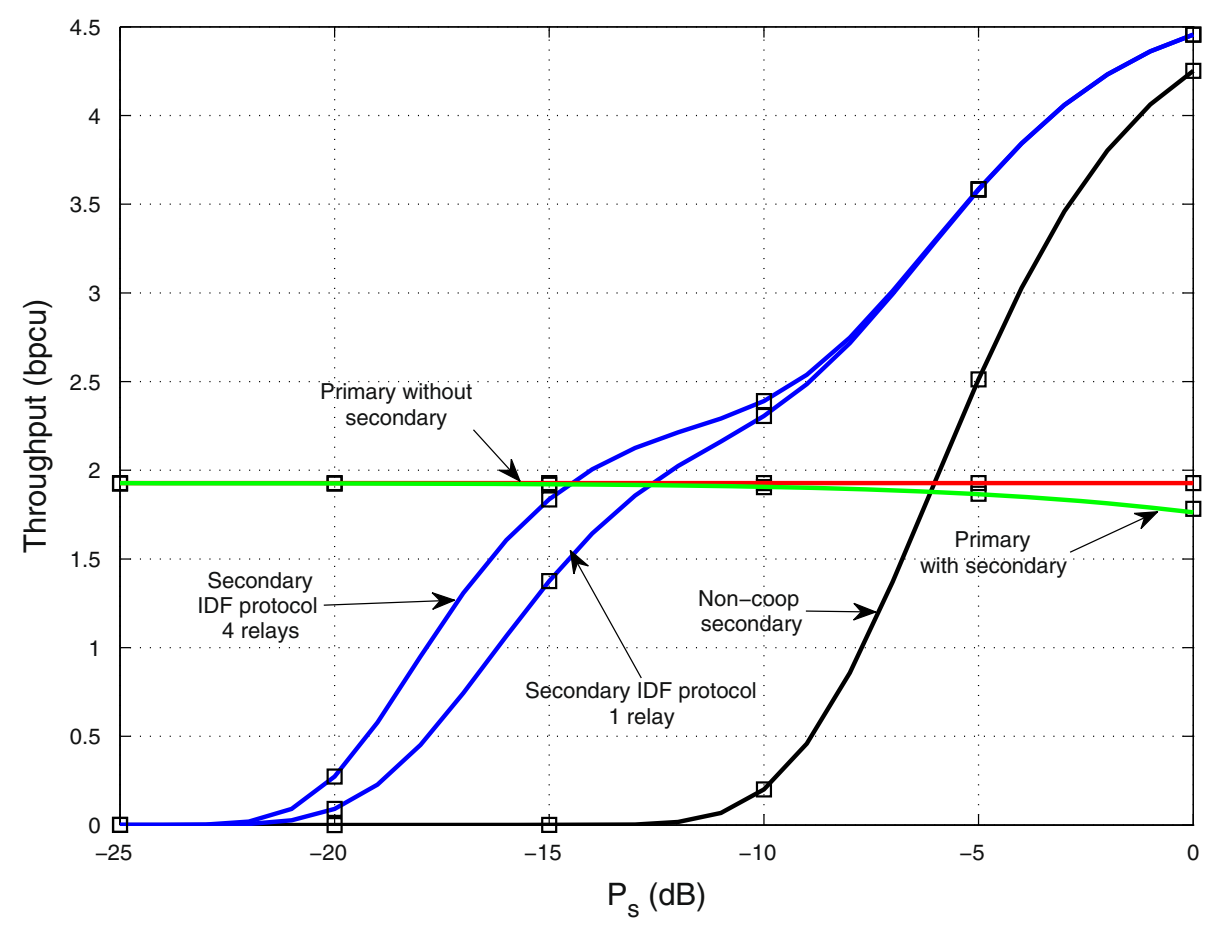

Figure 7 Throughput versus $P_{s}$, for $R_{p}=4 \mathrm{bpcu}, R_{s}=6 \mathrm{bpcu}, P_{p}=10 \mathrm{~dB}, \lambda_{p p}=\lambda_{s p}=\lambda_{r p}=1, \lambda_{p s}=\lambda_{p r}=4^{4}, \lambda_{s s}=4^{4}, \lambda_{s r}=\lambda_{r s}=8^{4}$, considering up to four available relays. The black square marker represents the Monte Carlo simulations. 


\section{Conclusions}

This paper introduces a new cooperative transmission scheme for overlay cognitive radio in which the secondary network exploits the primary retransmissions. We show that the throughput of the secondary network can be significantly increased with a very small performance loss imposed to the primary network. Selective and incremental decode-and-forward-based cooperative protocols were analysed as well as the impact of having multiple available relays. Our results show that the best configuration for the proposed scheme is when the secondary nodes are close to each other and nearby the primary transmitter, a situation in which the secondary network can achieve relatively high throughput without damaging the performance of the primary network.

\section{Appendices}

\section{Appendix 1 Probability of event $\boldsymbol{U}_{\boldsymbol{n}}$}

In order to write the probability of event $U_{n}$ (the event that no relay was able to correctly decode both the messages from $T_{p}$ and $T_{s}$ ), let us first define $\mathcal{O}_{s r(l)}$ and $\mathcal{O}_{p r(l)}$ as the outage probabilities of the $T_{s}$ to $r(l)$ and $T_{p}$ to $r(l)$ links. Then,

$$
\begin{aligned}
\mathcal{O}_{s r(l)} & =\operatorname{Pr}\left\{I_{s r(l)}<R_{s}\right\}=\operatorname{Pr}\left\{\log _{2}\left(1+\left|h_{s r(l)}\right|^{2} P_{s}\right)<R_{s}\right\} \\
& =\frac{\gamma\left(m_{s r(l)}, \frac{m_{s r(l)}\left(2^{R_{s}}-1\right)}{\lambda_{s r(l)} P_{s}}\right)}{\Gamma\left(m_{s r(l)}\right)}
\end{aligned}
$$

$$
\begin{aligned}
\mathcal{O}_{p r(l)}= & \operatorname{Pr}\left\{I_{p r(l)}<R_{p}\right\}=\operatorname{Pr}\left\{\log _{2}\left(1+\left|h_{p r(l)}\right|^{2} P_{p}\right)<R_{p}\right\} \\
= & \frac{\gamma\left(m_{p r(l)}, \frac{m_{p r(l)}\left(2^{R_{p}}-1\right)}{\lambda_{p r(l)} P_{p}}\right)}{\Gamma\left(m_{p r(l)}\right)} .
\end{aligned}
$$

Moreover, since there are $N$ available relays, then $\operatorname{Pr}\left\{U_{n}\right\}$ can be written as

$$
\begin{aligned}
\operatorname{Pr}\left\{U_{n}\right\} & =\left(1-\left(1-\mathcal{O}_{p r(l)}\right)\left(1-\mathcal{O}_{s r(l)}\right)\right)^{N} \\
& =\left(\mathcal{O}_{p r(l)}+\mathcal{O}_{s r(l)}-\mathcal{O}_{s r(l)} \cdot \mathcal{O}_{p r(l)}\right)^{N}
\end{aligned}
$$

The final form of $\operatorname{Pr}\left\{U_{n}\right\}$ as in Eq. (8) is attained by putting Eq. (15) and Eq. (16) into Eq. (17). Note that $\operatorname{Pr}\left\{U_{n}\right\}$ tends to zero as the number of relays $N$ increases.

\section{Appendix 2 Outage probability $\mathcal{O}_{p, 2}$}

First, let us write $\mathcal{O}_{p, 2}=\mathcal{O}_{p, 2}^{A}+\mathcal{O}_{p, 2}^{B}+\mathcal{O}_{p, 2}^{C}$, where

$$
\mathcal{O}_{p, 2}^{A}=\operatorname{Pr}\left\{I_{p, 2}<R_{p} \mid \overline{\mathcal{O}_{p s}}, \overline{U_{n}}\right\} \cdot \overline{\mathcal{O}_{p s}} \cdot \operatorname{Pr}\left\{\overline{U_{n}}\right\}
$$

$$
\begin{aligned}
& \mathcal{O}_{p, 2}^{B}=\operatorname{Pr}\left\{I_{p, 2}<R_{r} \mid \overline{\mathcal{O}_{p s}}, U_{n}\right\} \cdot \overline{\mathcal{O}_{p s}} \cdot \operatorname{Pr}\left\{U_{n}\right\}, \\
& \mathcal{O}_{p, 2}^{C}=\operatorname{Pr}\left\{I_{p, 2}<R_{p} \mid \mathcal{O}_{p s}\right\} \cdot \mathcal{O}_{p s} .
\end{aligned}
$$

Therefore, $\mathcal{O}_{p, 2}^{A}$ is

$$
\begin{aligned}
\mathcal{O}_{p, 2}^{A}= & \operatorname{Pr}\left\{\log _{2}\left(1+\left|h_{p p}\right|^{2} P_{p}\right)+\frac{1}{2} \log _{2}\left(1+\frac{\left|h_{p p}\right|^{2} P_{p}}{1+\left|h_{s p}\right|^{2} P_{s}}\right)\right. \\
& \left.+\frac{1}{2} \log _{2}\left(1+\frac{\left|h_{p p}\right|^{2} P_{p}}{1+\left|h_{r p}\right|^{2} P_{r}}\right)<R_{p}\right\} \cdot \overline{\mathcal{O}_{p s}} \cdot \operatorname{Pr}\left\{\overline{U_{n}}\right\} .
\end{aligned}
$$

In order to find a closed-form solution for Eq. (21), we consider an assumption already made in [29], in which it is considered the use of a whitening filter with the objective of converting the interference into an approximately Gaussian signal. A similar approach has also been considered in [8,30-32]. Thus, following this approach, we can consider the secondary interference as having a Gaussian distribution with zero mean and variance $\sigma_{r p}^{2}=$ $P_{r} \cdot \lambda_{r p}$, regarding the interference from the relay to the primary destination, and $\sigma_{s p}^{2}=P_{s} \cdot \lambda_{s p}$, regarding the interference from the secondary transmitter to the primary destination. Then, according to [29, Equation (7)], the overall Gaussian noise variance at the primary destination including the effect of the interference from the secondary transmitter is then $\sigma_{s}^{2}=\sigma_{n}^{2}+\sigma_{s p}^{2}$, while in the case of the interference coming from the secondary relay, we have $\sigma_{r}^{2}=\sigma_{n}^{2}+\sigma_{r p}^{2}$. Then, Eq. (21) can be rewritten as

$$
\begin{aligned}
\mathcal{O}_{p, 2}^{A} \approx & \operatorname{Pr}\left\{\log _{2}\left(1+\left|h_{p p}\right|^{2} P_{p}\right)+\frac{1}{2} \log _{2}\left(1+\frac{\left|h_{p p}\right|^{2} P_{p}}{\sigma_{s}^{2}}\right)\right. \\
& \left.+\frac{1}{2} \log _{2}\left(1+\frac{\left|h_{p p}\right|^{2} P_{p}}{\sigma_{r}^{2}}\right)<R_{p}\right\} \cdot \overline{\mathcal{O}_{p s}} \cdot \operatorname{Pr}\left\{\overline{U_{n}}\right\} \\
\leq & \operatorname{Pr}\left\{\log _{2}\left(1+\left|h_{p p}\right|^{2} P_{p}\right)+\frac{1}{2} \log _{2}\left(1+\frac{\left|h_{p p}\right|^{2} P_{p}}{\sigma_{p}^{2}}\right)\right. \\
& \left.+\frac{1}{2} \log _{2}\left(1+\frac{\left|h_{p p}\right|^{2} P_{p}}{\sigma_{p}^{2}}\right)<R_{p}\right\} \cdot \overline{\mathcal{O}_{p s}} \cdot \operatorname{Pr}\left\{\overline{U_{n}}\right\} \\
= & \operatorname{Pr}\left\{\log _{2}\left(1+\left|h_{p p}\right|^{2} P_{p}\right)+\log _{2}\left(1+\frac{\left|h_{p p}\right|^{2} P_{p}}{\sigma_{p}^{2}}\right)\right\} \\
& \cdot \overline{\mathcal{O}_{p s}} \cdot \operatorname{Pr}\left\{\overline{U_{n}}\right\},
\end{aligned}
$$

where $\sigma_{p}^{2}=\max \left[\sigma_{s}^{2}, \sigma_{r}^{2}\right]$. 
Then, the outage probability $\mathcal{O}_{p, 2}^{A}$ becomes

$$
\begin{aligned}
\mathcal{O}_{p, 2}^{A} \approx & \operatorname{Pr}\left\{\log _{2}\left(\left(1+\left|h_{p p}\right|^{2} P_{p}\right)\left(1+\frac{\left|h_{p p}\right|^{2} P_{p}}{\sigma_{p}^{2}}\right)\right)<R_{p}\right\} \cdot \overline{\mathcal{O}_{p s}} \cdot \operatorname{Pr}\left\{\overline{U_{n}}\right\} \\
\approx & \operatorname{Pr}\left\{\left(\left|h_{p p}\right|^{2}<\frac{\sqrt{\left(1+\sigma_{p}^{2}\right)^{2} \mid !+4 \sigma_{p}^{2} \cdot\left(2^{R_{p}}-1\right)}-1-\sigma_{p}^{2}}{2 P_{p}}\right)\right\} \\
& \cdot \frac{}{\mathcal{O}_{p s}} \cdot \operatorname{Pr}\left\{\overline{U_{n}}\right\} \\
\approx & \frac{\gamma\left(m_{p p}, m_{p p} \frac{\sqrt{\left(1+\sigma_{p}^{2}\right)^{2}+4 \sigma_{p}^{2} \cdot\left(2^{R p}-1\right)}-1-\sigma_{p}^{2}}{2 P_{p} \lambda_{p p}}\right)}{\Gamma\left(m_{p p}\right)} \cdot \overline{\mathcal{O}_{p s}} \cdot \operatorname{Pr}\left\{\overline{U_{n}}\right\} .
\end{aligned}
$$

Moreover, in order to write the second term, $\mathcal{O}_{p, 2}^{B}$, in closed form, we first note that

$$
\begin{aligned}
& \frac{1}{2} \log _{2}\left(1+\left|h_{p p}\right|^{2} P_{p}\right)+\frac{1}{2} \log _{2}\left(1+\frac{\left|h_{p p}\right|^{2} P_{p}}{1+\left|h_{s p}\right|^{2} P_{s}}\right) \\
& \quad \geq \log _{2}\left(1+\frac{\left|h_{p p}\right|^{2} P_{p}}{1+\left|h_{s p}\right|^{2} P_{s}}\right)
\end{aligned}
$$

as $\left|h_{s p}\right|^{2} P_{s} \geq 0$. Then,

$$
\begin{aligned}
& \mathcal{O}_{p, 2}^{B}=\operatorname{Pr}\left\{\log _{2}\left(1+\left|h_{p p}\right|^{2} P_{p}\right)+\frac{1}{2} \log _{2}\left(1+\left|h_{p p}\right|^{2} P_{p}\right)\right. \\
& \left.+\frac{1}{2} \log _{2}\left(1+\frac{\left|h_{p p}\right|^{2} P_{p}}{1+\left|h_{s p}\right|^{2} P_{s}}\right)<R_{p}\right\} \cdot \overline{\mathcal{O}_{p s}} \cdot \operatorname{Pr}\left\{U_{n}\right\} \\
& \leq \operatorname{Pr}\left\{\log _{2}\left(1+\left|h_{p p}\right|^{2} P_{p}\right)+\log _{2}\left(1+\frac{\left|h_{p p}\right|^{2} P_{p}}{1+\left|h_{s p}\right|^{2} P_{s}}\right)<R_{p}\right\} \\
& \cdot \overline{\mathcal{O}_{p s}} \cdot \operatorname{Pr}\left\{U_{n}\right\} \\
& \approx \operatorname{Pr}\left\{\log _{2}\left(1+\left|h_{p p}\right|^{2} P_{p}\right)+\log _{2}\left(1+\frac{\left|h_{p p}\right|^{2} P_{p}}{\sigma_{p}^{2}}\right)<R_{p}\right\} \\
& \cdot \overline{\mathcal{O}_{p s}} \cdot \operatorname{Pr}\left\{U_{n}\right\} \\
& =\frac{\gamma\left(m_{p p}, m_{p p} \frac{\sqrt{\left(1+\sigma_{p}^{2}\right)^{2}+4 \sigma_{p}^{2} \cdot\left(2^{R p}-1\right)}-1-\sigma_{p}^{2}}{2 P_{p} \lambda_{p p}}\right)}{\Gamma\left(m_{p p}\right)} \cdot \overline{\mathcal{O}_{p s}} \cdot \operatorname{Pr}\left\{U_{n}\right\} .
\end{aligned}
$$

The third term, $\mathcal{O}_{p, 2}^{C}$, can be simply written as

$$
\begin{aligned}
\mathcal{O}_{p, 2}^{C} & =\operatorname{Pr}\left\{\log _{2}\left(1+\left|h_{p p}\right|^{2} P_{p}\right)+\log _{2}\left(1+\left|h_{p p}\right|^{2} P_{p}\right)<R_{p}\right\} \cdot \mathcal{O}_{p s} \\
& =\frac{1}{\Gamma\left(m_{p p}\right)} \gamma\left(m_{p p}, \frac{m_{p p}\left(2^{\frac{1}{2} R_{p}}-1\right)}{\lambda_{p p} P_{p}}\right) \cdot \mathcal{O}_{p s}
\end{aligned}
$$

Finally, the complete outage probability $\mathcal{O}_{p, 2}$ is given by the sum of Eqs. (23), (25), and (26), as given in Eq. (9). The accuracy of the derived closed-form expressions is investigated in Section 4.

\section{Appendix 3 Outage probability $\mathcal{O}_{\text {MRC }}$}

The outage probability in Eq. (13),

$$
\operatorname{Pr}\left\{\log _{2}\left(1+\left(\left|h_{r s}\right|^{2}+\left|h_{s s}\right|^{2}\right) P_{s}\right)<R_{s}\right\},
$$

can be written in closed form for the case of some particular fading parameters. By defining $x=\left|h_{s s}\right|^{2}$ and $y=$ $\left|h_{r s}\right|^{2}$, and computing the distribution of the sum $z=x+y$ as $f_{z}(z)=\int_{0}^{z} f_{x}(z-y) \cdot f_{y}(y) d y$, where $f_{x}(x)=\frac{\exp \left(-\frac{x}{\lambda_{s s}}\right)}{\lambda_{s s}}$ and $f_{y}(y)=\frac{\exp \left(-\frac{y}{\lambda_{r s}}\right)}{\lambda_{r s}}$ are the probability density functions (pdf) of the variables $x$ and $y$ for the case NLOS, respectively. For instance, in the case of $m_{s r}=m_{s s}=1$ and $\lambda_{s s} \neq \lambda_{r s}$, in which all channels are in NLOS condition, we can show that

$$
\begin{aligned}
\mathcal{O}_{\mathrm{MRC}} & =\int_{0}^{\left(2^{R_{s}}-1\right) / P_{s}} f_{z}(z) d z \\
& =\frac{\lambda_{r s}-\lambda_{r s} \exp \left(-\frac{2^{R_{s}}-1}{\lambda_{r s} P_{s}}\right)-\lambda_{s s}+\lambda_{s s} \exp \left(-\frac{2^{R_{s}}-1}{\lambda_{s s} P_{s}}\right)}{\lambda_{r s}-\lambda_{s s}},
\end{aligned}
$$

while in the case of $m_{s r}=m_{s s}=2$, so that there are some LOS in the channels, following similar analysis as in the case of $m_{s r}=m_{s s}=1$ and considering $f_{x}(x)=\frac{4 \exp \left(-\frac{2 x}{\lambda_{s s}}\right) x}{\lambda_{s s}^{2}}$ and $f_{y}(y)=\frac{4 \exp \left(-\frac{2 y}{\lambda_{r s}}\right) y}{\lambda_{r s}^{2}}$, it is possible to write the outage probability as:

$$
\begin{aligned}
\mathcal{O}_{\mathrm{MRC}}= & \frac{1}{P_{s}\left(\lambda_{s s}-\lambda_{r s}\right)^{3}} \exp \left(-\frac{2\left(2^{R_{s}}-1\right)\left(\lambda_{s s}+\lambda_{r s}\right)}{P_{s} \lambda_{s s} \lambda_{r s}}\right) \\
& \times\left[\exp \left(\frac{2\left(2^{R_{s}}-1\right)\left(\lambda_{s s}+\lambda_{r s}\right)}{P_{s} \lambda_{s s} \lambda_{r s}}\right) P_{s}\left(\lambda_{s s}-\lambda_{r s}\right)^{3}\right. \\
& +\exp \left(\frac{2\left(2^{R_{s}}-1\right)}{P_{s} \lambda_{r s}}\right) \lambda_{s s}\left(2\left(2^{R_{s}}-1\right)\left(\lambda_{r s}-\lambda_{s s}\right)\right. \\
& \left.-P_{s} \lambda_{s s}\left(\lambda_{s s}-3 \lambda_{r s}\right)\right) \\
& +\exp \left(\frac{2\left(2^{R_{s}}-1\right)}{P_{s} \lambda_{s s}}\right) \lambda_{r s}\left(P_{s} \lambda_{r s}\left(\lambda_{r s}-3 \lambda_{s s}\right)\right. \\
& \left.\left.+2\left(2^{R_{s}}-1\right)\left(\lambda_{r s}-\lambda_{s s}\right)\right)\right]
\end{aligned}
$$

\section{Competing interests}

The authors declare that they have no competing interests.

\section{Acknowledgements}

This paper was partially presented at The Ninth International Symposium on Wireless Communication Systems (ISWCS), Paris, 2012. The authors would like to thank the support by CAPES and CNPq, Brazil, and Infotech Oulu, Finland.

\section{Author details}

${ }^{1}$ Federal University of Technology - Paraná (UTFPR), Curitiba, 80230-901 Paraná, Brazil. ${ }^{2}$ Electrical Engineering Department, Federal University of Paraná (UFPR), Curitiba, 81531-990 Paraná, Brazil. ${ }^{3}$ Centre for Wireless Communications (CWC), University of Oulu, PO Box 4500, 90014 Oulu, Finland. 
Received: 6 May 2013 Accepted: 10 July 2013

Published: 18 July 2013

\section{References}

1. J Mitola, GQ Maguire, Cognitive radio: making software radios more personal. IEEE Pers. Commun. 6(4), 13-18 (1999)

2. S Haykin, Cognitive radio: brain-empowered wireless communications. IEEE. J. Sel. Areas Commun. 23(2), 201-220 (2005)

3. A Goldsmith, S Jafar, I Maric, S Srinivasa, Breaking spectrum gridlock with cognitive radios: an information theoretic perspective. Proc. IEEE, 97(5), 894-914 (2009)

4. S Srinivasa, SA Jafar, The throughput potential of cognitive radio: a theoretical perspective. IEEE Commun. Mag. 45(5), 73-79 (2007)

5. DB da Costa, S Aissa, CC Cavalcante, Performance analysis of partial relay selection in cooperative spectrum sharing systems. Wireless Person. Commun. 64(1), 79-92 (2012)

6. TQ Duong, DB da Costa, TA Tsiftsis, C Zhong, A Nallanathan, Outage and diversity of cognitive relaying systems under spectrum sharing environments in Nakagami-m fading. IEEE Commun. Lett. 16(12), 2075-2078 (2012)

7. TQ Duong, DB da Costa, M Elkashlan, VN QBao, Cognitive amplify-and-forward relay networks over Nakagami-m Fading. IEEE Trans. Veh. Techol. 61(5), 2368-2374 (2012)

8. A Jovicic, P Viswanath, Cognitive radio: an information theoretic perspective. IEEE Trans. Inf. Theory. 55(9), 3945-3958 (2009)

9. RC Pereira, RD Souza, ME Pellenz, Multiple concurrent transmissions in wireless mesh networks employing superposition and dirty paper coding. IEEE Trans. Veh. Technol. 58(9), 5115-5123 (2009)

10. RA Tannious, A Nosratinia, Cognitive radio protocols based on exploiting hybrid ARQ retransmissions. IEEE Trans. Wireless Commun. 9(9), 2833-2841 (2010)

11. RD Souza, Reducing co-existence penalty of retransmission-based cognitive radio protocol. IEE Electron. Lett. 47(6), 409-411 (2011)

12. JN Laneman, DNC Tse, GW Wornell, Cooperative diversity in wireless networks: efficient protocols and outage behavior. IEEE Trans. Inf. Theory 50(12), 3062-3080 (2004)

13. A Sendonaris, E Erkip, B Aazhang, User cooperation diversity. Part I. System description. IEEE Trans. Commun. 51(11), 1927-1938 (2003)

14. A Nosratinia, TE Hunter, A Hedayat, Cooperative communication in wireless networks. IEEE Commun. Mag. 42(10), 74-80 (2004)

15. A Bletsas, A Khisti, DP Reed, A Lippman, A simple cooperative diversity method based network path selection. IEEE J. Select. Areas Commun. 24, 659-672 (2006)

16. I Krikidis, J Thompson, JS McLaughlin, N Goertz, Amplify-and-forward with partial relay selection. IEEE Commun. Letters. 12(4), 235-237 (2008)

17. TQ Duong, H Zepernick, Hybrid decode-amplify-forward cooperative communications with multiple relays, in IEEE Wireless Communications and Networking Conference (Budapest, 5-8 April 2009), pp. 1-6

18. DB da Costa, S Aissa, Performance analysis of relay selection techniques with clustered fixed-gain relays. IEEE Signal Proc. Lett. 17(2), 201-204 (2010)

19. I Krikidis, T Charalambous, JS Thompson, Buffer-aided relay selection for cooperative diversity systems without delay constraints. IEEE Trans. Wireless Commun. 11(5), 1957-1967 (2012)

20. NS Ferdinand, U Jayasinghe, N Rajatheva, M Latva-aho, Impact of antenna correlation on the performance of partial relay selection. EURASIP J Wireless Commun. Netw. 2012, 1-13 (2012)

21. N Nakagami, The m-distribution, a general formula for intensity distribution of rapid fading, in Statistical Methods in Radio Wave Propagation, ed. by W. G. Hoffman (Pergamon,Oxford, 1960)

22. MD Yacoub, The $\kappa-\mu$ distribution and the $\eta-\mu$ distribution. IEEE Antennas Propagation Mag. 49(1), 68-81 (2007)

23. A Goldsmith, Wireless Communications (Cambridge University Press, New York, NY, 2005)

24. R Knopp, P Humblet, On coding for block fading channels. IEEE Trans. Inf. Theory 46(1), 189-205 (2000)

25. E Biglieri, G Caire, G Taricco, Limiting performance of block-fading channels with multiple antennas. IEEE Trans. Inf. Theory 47(4), 1273-1289 (2001)
26. MHM Costa, Writing on dirty paper (Corresp.) IEEE Trans. Inf. Theory 29(3), 439-441 (1983)

27. Z Wang, G Giannakis, A simple and general parameterization quantifying performance in fading channels. IEEE Trans. Commun. 51(8), 1389-1398 (2003)

28. S Savazzi, U Spagnolini, Cooperative space-time coded transmissions in Nakagami-m fading channels, in IEEE Global Telecommunications Conference (Washington, DC, 26-30 November 2007), pp. 4334-4338

29. H Kim, S Lim, H Wang, D Hong, Optimal power allocation and outage analysis for cognitive full duplex relay systems. IEEE Trans. Wireless Commun. 11(10), 3754-3765 (2012)

30. A Punchihewa, VK Bhargava, C Despins, Capacity and power allocation for cognitive MAC with imperfect channel estimation. IEEE Trans. Wireless Commun. 10(12), 4001-4007 (2011)

31. E Pei, S Wang, Z Zhang, Capacity and optimal power allocation for spectrum-sharing with primary transmission consideration in fading channels. IEEE Commun. Lett. 15(4), 389-391 (2011)

32. X Gong, SA Vorobyov, C Tellambura, Optimal bandwidth and power allocation for sum ergodic capacity under fading channels in cognitive radio networks. IEEE Trans. Signal Proc. 59(4), 1814-1826 (2011)

\section{doi:10.1186/1687-1499-2013-196}

Cite this article as: Mafra et al:: Cooperative overlay secondary transmissions exploiting primary retransmissions. EURASIP Journal on Wireless Communications and Networking 2013 2013:196.

\section{Submit your manuscript to a SpringerOpen ${ }^{\circ}$ journal and benefit from:}

- Convenient online submission

- Rigorous peer review

- Immediate publication on acceptance

- Open access: articles freely available online

- High visibility within the field

- Retaining the copyright to your article

Submit your next manuscript at $\gg$ springeropen.com 